\title{
SABERES, PODERES Y PRACTICAS SOCIALES
}

\author{
Diego Jaramillo Salgado \\ Universidad del Cauca, Popayán, Colombia
}

\section{INTRODUCCIÓN.}

Este trabajo se hace desde la filosofía, pero no está pensado ni realizado en función estricta de la comunidad académica de los filósofos; lo cual no le resta pertinencia en relación con la disciplina filosófica. Al contrario, plantea una forma de interlocución de esta disciplina con otras de las ciencias sociales en campos de epistemologías y de teorías del conocimiento. Esto indica que se asumen diferencias con las denominadas ciencias naturales; lo cual no significa que se excluyan elementos conceptuales que puedan ser aplicables a toda práctica de conocimiento o a toda práctica científica. De hecho, los análisis se harán con prioridad sobre los problemas que se puedan considerar de mayor énfasis en las disciplinas y ciencias sociales.

Esta especificidad es tratada en el estudio del carácter de las disciplinas y de las ciencias sociales en lo relacionado con la aproximación a la realidad que efectúa el sujeto en la pretensión de conocer; por tanto, la construcción que hace de ella y el proceso de formación del sujeto de conocimiento. Este se asume como un ser social inmerso, de una u otra forma, en las mismas circunstancias en que se produce lo que conoce o que es objeto de investigación, o en las relaciones de determinación que posee lo que va a investigar. De la misma manera, se trata de tener en cuenta lo que se denomina objeto de investigación u objeto de conocimiento como constituido por problemas en los que se encuentra implicado el hombre; es decir, inscrito en practicas culturales, simbólicas, políticas, sociales, etc. En particular nos interesa acentuar la relación poder-saber como elemento explicativo de la propia formación de los discursos y de su incidencia en las prácticas sociales.

Por último, nos detendremos en una aproximación a las implicaciones pedagógicas que podría tener una reflexión de este tipo. Se trata de aventurar opciones que posibiliten incentivos a la creatividad y a la búsqueda de rompimientos de lo que Kant llamara la "minoría de edad", y Paulo Freire, la "educación bancaria". 


\section{EL SUJETO DE CONOCIMIENTO.}

El sujeto que conoce, que investiga, en cuanto ser social, es portador de ideologías, de representaciones sociales, de imaginarios sociales por medio de los cuales anticipa prejuicios y preconceptos de la realidad que va investigar. Se aproxima a partidos políticos, doctrinas religiosas o morales. Se inscribe dentro de una cultura y de una tradición cultural. Tiene una procedencia de clase social, grupo social y familia o una historia individual. Es partícipe de las relaciones de fuerza que se producen en la sociedad, y no puede escapar al conflicto de los intereses en que se inscribe su práctica social. De tal manera que el nivel de "cientificidad" estará matizado por las intersecciones de diferentes prácticas sociales.

A la vez, el sujeto de conocimiento valida una conceptualización con la cual se propone realizar el análisis e interpretación de la realidad. Pero, en el caso de las ciencias sociales, es factible admitir que no se pueden utilizar teorías con el propósito de universalizarlas o absolutizarlas, haciéndolas totalizantes. De tal manera que las corrientes teóricas, escuelas o autores que se les considere básicos pueden aproximarse o relacionarse entre sí porque se aproximan en algunos conceptos y tesis de igual significado; pero se pueden distanciar en muchos de ellos, llegando incluso a constituir postulados opuestos.

Si esto se afirma de los elementos que entran en relación con la producción de conocimientos, también se debe hacer con sus resultados. Sólo que éstos se desvinculan de su "autor", el sujeto que conoce, el sujeto que investiga. Adquieren una autonomía y se inscriben dentro de la disciplina o disciplinas en que se desarrolla ese saber; incorporando los aspectos de cientificidad o de universalidad que se les asigna en ellas.

\section{LA PRODUCCION DE CONOCIMIENTOS ES UNA PRACTICA SOCIAL}

Los fundamentos epistemológicos de las ciencias sociales involucran el sentido que puede tener el conocimiento como practica social. De tal manera que todo proceso de conocimiento está determinado por múltiples prácticas sociales y, al mismo tiempo, sus resultados pueden incidir en aquellas que se expresan dentro de la sociedad.

La práctica social incluye las disímiles actividades del hombre, en el sentido de su relación inmediata con el mundo que le rodea, y el sistema social y la sociedad en que se encuentra. Abarca su pasión, sus deseos, sus 
sentimientos. Su mundo familiar, la escuela, las instituciones, el trabajo. Su relación individual, su pertenencia de clase, su vinculación a grupos de determinado interés, etc.

También incluye la racionalidad que se ha producido sobre esas actividades. Filosóficamente hablan- do, es la vinculación teoría-práctica. Es una relación que, a la par, sitúa y clasifica los saberes. La especificidad de cada formación teórica identifica un tipo de práctica social, de disciplina o de ciencia, en lo que se refiere a las ciencias sociales.

No podría ser excluida de estas reflexiones la referencia al poder, en cuanto que toda práctica social está atravesada por relaciones de poder. Tradicionalmente se ha circunscrito la esfera del poder a lo que tiene que ver con el Estado y su relación con las clases sociales; de una manera "macrocefálica". Sin embargo, es necesario tener en cuenta que en la formación de un discurso o en su interiorización se induce o se asume de hecho la exclusión de otros. Los círculos de saber se organizan como sociedades en las cuales se hace más evidente la legitimación de ciertas formas de poder. Fácilmente se puede hacer la homologación a las que entre nosotros se denominan comunidades académicas o comunidades científicas. El fundamento de verdad que se produce desde la conceptualización, desde su misma enunciación, es por sí mismo una instancia de poder. No solo se refiere a la verbalización, en cuanto la verdad se enuncia y se forma en el lenguaje, y, como dijera Barthes:

"... El lenguaje es una legislación, la lengua es su código. No vemos el poder que hay en la lengua porque olvidamos que toda lengua es una clasificación y que toda clasificación es opresiva...".

Lo cual no puede llevar a concluir que el atributo de poder se lo asigne su adscripción a una normatividad, su inclusión dentro de una clasificación o el carácter negativo de una forma de imposición. Se trata de los nexos que establecen entre sí quienes se ubican dentro de códigos que solo ellos conocen, o que hegemónicamente controlan; igualmente, la fuerza que adquiere al afirmarse como saber. ${ }^{2}$ De esta manera, no se refiere exclusivamente a los saberes que se sitúan en formalidades discursivas científicas sino también a aquellos que se diseminan en las diferentes prácticas sociales.

Los saberes no se reducen a un resultado de la aplicación de formas lógicas del conocimiento; sino que atraviesan y son atravesados por el cuerpo, los deseos, las cosas, la cultura, etc. De ahí la inmanencia del poder. Lo afirma Godelier:

1 Roland Barthes. Lección inaugural. Ed. Siglo XXI, México, 1984, p. 118.

2 Maurice Godelier. “Poder y Lenguaje”, en Revista Eco, No. 247, Bogotá, 1982, p.90. 
"Poder y lenguaje están en una relación que no es solamente la marca en el lenguaje de una diferencia, Es más esencialmente un acceso, a través del lenguaje, a la esencia oculta de las cosas, Es un poder sobre las cosas, y, por consiguiente, un poder sobre los hombres a través del poder sobre las cosas".

La relación del poder con el saber es recíprocamente equivalente. Si hay efectos de poder en el saber; al mismo tiempo, el poder es expresado por medio de un saber y es en sí mismo poder discursivo.

De esta manera, el universo de esta práctica social no queda reducido a lo que Althusser denominó "práctica teórica". No se le circunscribe al particular ejercicio racional sino que tiene en cuenta los ejes de relaciones en los que se inscriben quien produce conocimientos y los fragmentos de realidad que se van a conocer. La práctica teórica -si se persiste en llamarla así- adquiere un específico significado si se la toma como práctica social. Además, no se ubica tampoco en la confrontación de la verdad con el error, tal como lo había establecido el filósofo francés. Más bien se rescata la noción foucultiana de "saber", en la que toda verdad, todo resultado de un discurso científico hay que analizarlo en su proceso de formación, incluyendo en él al error, la falsedad, la ignorancia; herencia que provenía desde el mismo Spinoza.

Es factible admitir que al saber no se le puede identificar con una exclusiva forma racional. Es decir, con la elaboración lógica de los conceptos o las categorías y la coherencia de una argumentación. Se puede entender, más bien, como parte de la vida misma. Inscrito en las relaciones de poder. Atravesado por los imaginarios sociales constituido por procesos culturales. Inmerso en el lenguaje, que, ajuicio de Barthes, es "aquél objeto en el que se inscribe el poder desde toda la eternidad humana". ${ }^{4}$

Situados aquí se podría calificar de nihilista o de escéptica la reflexión sobre el conocimiento. De una parte, el objeto se esfuma. Hay un devenir que, aparentemente, lo hace inaprensible. De otra, el sujeto se encuentra dentro de una realidad de la cual no puede ser ajeno: lo construye y a la vez es construido por él. Sin embargo, no se trata de establecer un relativismo tal que anule la conceptualización sino de hacer una reflexión que tenga como fundamento la crítica y como tal ubique relaciones y sentidos del conocimiento.

3 Ibidem. p. 90

4 Roland Barthes. Op. Cit. p. 118 


\section{EL METODO. LA RELACION “SUJETO - OBJETO”.}

En principio, se puede relativizar el sentido de la delimitación de lo que se denomina objeto de conocimiento. Cabe preguntarse: es posible definir los contornos de lo que se va a investigar? Se puede dar una respuesta afirmativa, si por ello se entiende el eje de relaciones en el cual se propone circunscribir un pro- ceso investigativo o de conocimiento. También puede ser negativa si se acepta que el llamado "objeto" de las ciencias sociales es cambiante y el mismo sujeto es objeto de cambio.

Por ello, al tratar de constituirlo se debieran cuestionar los elementos con los cuales se intenta hacerlo. Por ejemplo, en el estudio de la familia, el acercamiento a la definición de un objeto incluye aspectos jurídicos, sicológicos, sociológicos, la corriente o corrientes del pensamiento en las cuales se inscribe. Si es desde el derecho se inducirá la investigación hacia el derecho de familia, paternidad responsable, divorcio, patrimonio familiar, etc. Si es desde enfermería o medicina se elegirán factores genéticos causales de enfermedades, formas de prevención de enfermedades, etc. Si es desde la sociología, se podrá analizar a la familia como grupo social y sus relaciones con otros grupos; si es desde la antropología, el machismo, la forma matriarcal o patriarcal, las relaciones de parentesco, etc. Fuera de ello, se deben tener en cuenta las características propias de los grupos sociales o culturales a los cuales se realizará la investigación. Al mismo tiempo, en cada elección se incluirán aspectos teóricos que pueden llevar a conclusiones diferentes.

Esto permite concluir que "la familia", como objeto, no es más que un tema o problema para investigar; pero no es el objeto en el sentido epistemológico. Porque cuando se trata de definirlo, se hace en una intersección de elementos que implican una construcción. No se trata, por tanto, del dato empírico bruto. No es algo que senos revela, que está allí esperándonos para poder asirlo. Se trata de un ordenamiento que está dado desde la subjetividad. Por supuesto, una subjetividad definida históricamente.

Se puede encontrar un respaldo a esta afirmación en Kant, especialmente en el fundamento racional, cuando afirma que si "Galileo hizo rodar sobre un plano inclinado las bolas cuyo peso había señalado... se comprendió que la razón solo descubre lo que ella ha producido según sus propios planes".5 Refiriéndose a que el proceso del conocimiento científico no parte necesariamente del hecho en cuanto tal, como lo asentían Bacon y Locke, entre otros, sino que hay una preconstrucción por medio de la cual se estructura el objeto de conocimiento.

5 Kant. Crítica de la razón pura. p. 130 
Igualmente se puede recurrir a Elí de Gortari, cuya producción teórica está inscrita dentro del marxismo; por lo tanto difiere de Kant en reconocerse materialista, pero afirma: “... para emprender cualquier investigación concreta se parte siempre de varios supuestos primordiales... formados por los postulados de la disciplina de que se trate, los postulados del grupo de ciencias a que pertenezca y los postulados del conocimiento científico en general". 6 Solo que estos autores, acorralados de una u otra forma por la racionalidad, dejan de lado todo lo que tiene que ver con lo irracional, el inconsciente, los deseos, la cultura, lo simbólico, etc.

En conclusión, es posible aceptar que la formación del objeto de conocimiento se efectúa, en una primera instancia con preconceptos y con elementos de la vida cultural e ideológica. De allí que se tenga que partir de la crítica, como lo planteaba Marx. No sólo se critica la realidad sino, principalmente, el conocimiento anterior de la ciencia, la disciplina o los saberes que le son pertinentes. Ya sea para legitimarlos, corroborando sus aciertos, para establecer sus carencias o vacíos o para definir el nuevo rumbo que se debe tomar en el campo del saber que se cuestiona y de los problemas que se proyectan resolver.

La existencia de esos preconceptos y prejuicios no se imponen al objeto condicionándolos en su totalidad a la voluntad del sujeto. Si, en efecto, hay una cierta construcción de la realidad, ella se ubica dentro procesos que incluyen tanto la relación recíproca entre los fenómenos, los hechos y las cosas, como la interrelación que se produce entre el sujeto y el objeto en el proceso del conocimiento. Así, no puede entenderse que la "actividad" del sujeto se produzca en una relación de exterioridad al objeto mismo sino que se realiza en la dinámica de producción de las categorías y conceptos que Hegel y Marx denominaron concreto de pensamiento. Además, tiene en cuenta la incidencia que este proceso puede producir en el propio objeto. De ahí que sea tan importante tener en cuenta la Tesis III de Marx sobre Feuerbach:

"La teoría materialista de que los hombres son producto de las circunstancias y de la educación, y de que, por tanto, los hombres modificados son producto de circunstancias distintas y de una educación modificada, olvida que son los hombres, precisamente, los que hacen que cambien las circunstancias y que el propio educador necesita ser educado."

Si se reconoce que hay un proceso propio del pensamiento en la producción de conocimiento y se resalta una cierta disposición apriorística, no es para anular la interrelación que se establece entre sujeto y realidad. La cita de Marx es clara; la determinación material no se entiende como el reconocimiento de una pasividad del sujeto. A ello se le agrega la contraparte 6 Eli de Gortari. Lógica general. p. 34-35

7 Federico Engels. 11 tesis sobre Feurbach. Ediciones en lenguas extranjeras, Pekín, 1974. p.2 
dialéctica, o sea la capacidad del hombre de cambiar las circunstancias. En relación con las ciencias socia- les, esto es mucho más significativo porque los resultados de la investigación pueden establecer un nuevo ordenamiento de la realidad o coadyuvar a transformarla radicalmente. Este argumento puede también ser base de la explicación del desarrollo de las ciencias y de las disciplinas y del surgimiento y formación de otras.

Entre los elementos previos y constitutivos del proceso del conocimiento se encuentran las ideologías. Factor básico de mediación y de indicación de sentidos. Problema que fue abordado desde diferentes orientaciones en las dos décadas pasadas, de las cuales es necesario presentar las direcciones generales que se ubicaron.

Una de ellas es la que desarrolla Althusser. Retorna a Marx, quien define la ideología como error, como falsedad, como mixtificadora de la realidad. Los su- puestos de esta discusión se produjeron como resulta- do de la crítica a Hegel y a toda la filosofía anterior. Ya fuera idealista o materialista, Marx situaba a toda la filosofía que le antecedió a su pensamiento como metafísica y encubridora de la realidad. Por ello concluía que hasta él, la filosofía sólo se había ocupado de interpretar el mundo y de lo que se trataba era de transformarlo. Fue asumida por el francés en esta dirección con su consecuente opuesto, la ciencia sus- tentada en la verdad y con la solución política de una sociedad científica.

"Otra es la que se argumenta como concepción del mundo. O sea un campo más amplio en el que no necesariamente se circunscribe a la función mixtificadora. También se diferencia de la anterior, porque aquella se le asignaba a la clase explotadora. Esta abarca a todas las clases y a las acciones de los individuos. Ambas pueden estar atravesadas por las contradicciones de clase, pero esta última no se reduce a ello y se sitúa en un espectro de más amplitud. Esto hace que tanto a nivel de grupo, de clase o de individuo se pueda tener una forma dominante de la ideología y tener otra contraria en muchos aspectos de la vida social. Es en esta forma como aparecen involucradas las instituciones y las representaciones, las diferentes formas de la cultura y las múltiples expresiones de la irracionalidad y las ilusiones.

Se ha llegado incluso a plantear la existencia de una ideología científica. Destacando con ello la extensión, que muchas veces se pretende hacer, de los postulados de las ciencias a todas las prácticas sociales. Define el propósito de racionalizar la vida, de atravesarla por el tamiz de las leyes científicas.

Cualquier orientación, de las anteriormente señaladas, que se asuma, en lo concerniente a las ideologías, se articula claramente con los problemas de formación de los conocimientos que se han analizado hasta ahora. Por tanto, 
marcan ejes de relación en que se sitúa un discurso sobre el conocimiento. Sin embargo, debe aceptarse como una de las tantas posibilidades en que se produce cualquier proceso del conocimiento. Aclaración que permite hacer mención a otros aspectos inscritos dentro de diferentes prácticas sociales, como la formación de las representaciones sociales y de los imaginarios sociales. En ellos la ideología está presente al igual que los sentimientos, los deseos, las prácticas simbólicas. De tal manera que se rompe la relación dicotómica entre ciencia e ideología y la determinación o prevalecía la establece las propias condiciones en las cuales se inscribe la realidad que se construye.

\section{EL RESULTADO DEL CONOCIMIENTO}

Si se parte de la filosofía hegeliana o de la marxista, se ha de considerar tanto el devenir de la realidad como el del conocimiento. Esto explica que cambie el segmento de realidad adoptado como objeto de conocimiento y que solo se pueda aprehender un eje de relaciones claramente establecido. Así mismo, implica la relativización de la acción del sujeto de conocimiento. Ello introduce una transitoriedad en los resultados, pero le asigna, a su vez, la especificidad a cada práctica de conocimiento. Todo está en devenir, pero cada práctica, cada cosa, cada segmento de realidad contiene formas particulares de existencia del movimiento. Todo acontecimiento histórico, toda acción social, todo práctica ideológica es definida desde un proceso que le es particular.

El sentido que aquí se presenta es el de la adopción de la categoría proceso. Por medio de ella podemos incluir aquello que es propio de la existencia universal de los seres: el carácter dialéctico de su devenir. A la vez, lo que singulariza a cada elemento en particular.

Fuera de ello, permite ubicar las diferentes etapas en que se efectúa el conocimiento. Por tanto, no se detiene únicamente en antecedentes y consecuentes, en causas y efectos, en comienzos y resultados, integra todas las contradicciones, los desplazamientos, las fases, los elementos de cambio que recorren o que se hayan dado de principio a fin. Así se diferencian las disciplinas y las ciencias, los sujetos y objetos de conocimiento. Así se establecen también los ejes de relación que pueden presentarse entre sí.

Por ello, también se puede entender que a la verdad no se llega a través de un camino depurado, sin ninguna contaminación. Ya se ha planteado que son muchos los aspectos que intervienen en la producción de conocimientos. Se presentan tanto como efectos sociales o como elementos individuales, que a su vez también son sociales. En este sentido, como práctica de cono- cimiento, interviene también el error. No como negación del conocimiento sino 
como forma de conocimiento. Puede ser una limitante para la producción de una verdad, pero también puede ser un coadyuvante en esa vía, si se identifica en el proceso para su superación. Además, si un saber se inscribe dentro de un contexto cultural en el cual se produjo, se puede L llegar a encontrar que lo que para una cultura es verdad para otra puede ser error. $\mathrm{O}$ ni lo uno ni lo otro sino que se inscribe dentro de esas prácticas culturales con un efecto social o del lenguaje en el cual adquiere validez.

En la misma dirección se ubica la ignorancia. No se nace aprendido. No se conoce todo al nacer. Tampoco la mente es una tabula rasa, como la definía Locke. Los discursos, los saberes se producen y reproducen dentro de una práctica social. De ella hace parte la ignorancia que ha sido calificada como "vacío" y "carencia” de conocimientos. Estanislao Zuleta demostró que, por el contrario es una "llenura" de conocimientos que se tiene que criticar. Por tanto, se parte del proceso de conocimiento. Desde el punto de vista pedagógico, como se verá más adelante, es importante poder fundamentar la ignorancia, en términos de saber definir qué es lo que no se sabe y qué lo que se propone conocer. Con esto se rompe un sentido del positivismo de pretender hacer aparecer siempre la verdad única- mente como resultado, sin tener en cuenta el papel del error, la falsedad y la ignorancia en el proceso de su constitución.

Es en esta forma como se llega a un resultado que es la verdad o el "concreto de pensamiento", como lo denomina Marx, quien, a su vez, lo había tomado de Hegel. La diferencia entre ambos está en el orden de determinación. Uno desde los procesos materiales, otro desde los racionales. Pero ambos coinciden en que la producción de conocimientos es propia del pensamiento y que las "reglas" de la razón son las que lo legitiman. Ambos superan el mecanicismo anterior. El del empirismo, que hace del sujeto un ser contemplativo de la realidad exterior; reduciendo su actividad a fotografiar el mundo que se le presenta. El del racionalismo anterior a Kant, y, en parte, el de Kant mismo, que fundamenta el conocimiento en un apriorismo, basado en principios que se le imponen a la realidad o a lo que es objeto de conocimiento, sin tener en cuenta, estas dos corrientes del pensamiento, que "yo siento y pienso, no como un sujeto opuesto al objeto sino como un sujeto-objeto, como un ser real material ... el mundo objetivo no se encuentra solamente fuera de mí está también en mí mismo, en mi propia piel".!8

La verdad se expresa por medio de conceptos, axiomas, leyes, tesis, y en cuanto resultado se define como "síntesis de múltiples determinaciones". Esto

8 George Plejanov. Las cuestiones fundamentales del marxismo. Editor Rojo, Bogotá, 1975, p. 19. 
hace que lo que inicialmente se presentaba como algo caótico, indeterminable, indefinible, ahora se expresa bajo la forma ordenada y sistemática que le impone el pensamiento.

Allí sí se cumple lo que Gortari califica como el fin de la investigación: descubrir "las relaciones y propiedades que se mantengan relativamente constantes"; lo cual significa también que el objeto o, más precisa- mente, el tipo de "realidad" que se captura, nunca se agota y lo que se aprehende es aquél núcleo de relaciones que se propuso investigar; aquellas que produjo el mismo proceso de investigación y los sentidos que se encuentran en él.

Por ello, la verdad no es copia de la realidad. No es un espejo que refleja la realidad tal como ella es. Tampoco es la realidad. Además, "no se puede partir sosteniendo que se produzca una "correspondencia" entre lo real y lo racional, pues lo racional no refleja aquél sino que lo reconstruye". 9 Es por eso que Marx diferencia el concreto del pensamiento, como resulta- do del proceso del pensamiento, del concreto real, de lo que es, de lo que existe independientemente del sujeto que piensa, que percibe o que siente. Argumento que, en este aspecto, también tomó Althusser, haciendo un rastreo desde Spinoza y lo expresó bajo la figura: la idea de perro no ladra.

La teoría, al aceptarse como propia da un resultado que le fue previo se convierte en una guía para la acción o un elemento que posibilita la transformación de esa realidad. La teoría tiene su confrontación en la práctica; no entendida como la forma empírica del activismo sino de las posibilidades de contratación de los resultados teóricos con la realidad; o sea, que no basta la validez lógica que pueda tener una verdad. Además, es necesario registrar que el resultado de la investigación se independiza de su autor y puede ser apropiado o utilizado por cualquiera. "Las herramientas teóricas no tienen fronteras históricas o geográficas. La teoría es única... se trata también de recordar que es necesario producir constantemente nuevos conceptos, descubrir nuevas leyes a medida que las condiciones históricas cambian". ${ }^{10}$ Lo cual hace que la universalidad del pensamiento se articule con la particularidad de los acontecimientos reales, demostrando su especificidad, y ésta, a su vez, cuestione la universalidad produciendo otros universales.

9 Hugo Zemelman. Historia y politica en el conocimiento. UNAM, México, D.F, 1983,p.35 10 Manuel Castells. La cuestión urbana. Editorial siglo xxi, México, 1978. Introducción p. xi. 
Diego Jaramillo Salgado. Saberes, poderes y prácticas sociales.

\section{EL AZAR DEL PROCESO PEDAGOGICO}

Al llegar a este punto es necesario pensar las implicaciones pedagógicas que producen estas reflexiones epistemológicas. Sobre todo, si se inscribe en lo que Bachelard llamó "espíritu científico" o en una orientación que se puede corresponder más con lo expuesto; la formación de un pensamiento crítico o espíritu crítico.

Se trata de propiciar la salida de la que Kant denominó la minoría de edad. Aquella condición en que se quiere hacer depender a los individuos, en este caso a los estudiantes, de quien cree poseer toda la sabiduría: el profesor, o el tutor; "los tutores, que tan bondadosamente se han arrogado este oficio, cuidan muy bien que la gran mayoría de los hombres, (y no digamos que todo el sexo bello) considere el paso de la emancipación, además, de muy difícil, en extremo peligroso". ${ }^{11} \mathrm{Al}$ mismo tiempo, se hace extensivo también a la confrontación al dogmatismo. En la orientación kantiana se tiene como fundamento para ello la formación racional en una doble implicación: fundamentos de la verdad en la formación de las ciencias y conciencia de la libertad en la vida del ciudadano. Lo cual en términos de Estanislao Zuleta se sintetiza en tres principios, en relación con lo que se viene trabajando: "1.Pensar por sí mismo. 2. Pensar en el lugar del otro. 3. Ser consecuente". ${ }^{12}$

En el proceso de la práctica pedagógica se puede incentivar una formación crítica si se confronta la actividad repetidora, transmisora. Si se supera la reducción al dato, al hecho, a la descripción o al estricto conocimiento del diccionario. Si se critican los conocimientos generales, superficiales. Si no se reduce la actividad pedagógica a los resultados del conocimiento, sin tener en cuenta su proceso. Se intenta reivindicar la importancia de la práctica pedagógica centrada en un seguimiento al proceso de producción de una verdad o a los elementos que entraron en juego para la formación de un discurso científico o para la constitución de un saber determinado.

Esta última dirección fue trabajada y propuesta, entre otros, por Estanislao Zuleta en relación con la filosofía, refiriéndose a Platón; Elí de Gortari, en relación con la enseñanza de las ciencias naturales y Federico Sciacca, en la relación educación-filosofía, refiriéndose prioritariamente a la educación. A través de ellos, se puede concluir que una pedagogía creativa no constata ni memoriza resultados sino, que investiga o estudia el proceso de conocimiento que posibilitó la producción de una verdad, un concepto,

11 Kant. “QQué es la ilustración?”, en Filosofía de la historia. FCE, México, D.F., 1978, p. 26 12 Estanislao Zuleta. "Democracia y participación en Colombia” en Revista Foro, No. 6, Bogotá, 1988, p. 105 
una categoría, un saber. Esto hace del mismo docente un investigador en tanto que también él sigue ese proceso y va descubriendo tanto la configuración de las verdades, como los "vacíos" o las líneas de investigación que quedan insinuadas o los problemas que no quedan resueltos. Es sugestivo y sugerente, por lo menos, que la reflexión sobre la pedagogía desde estas tres disciplinas induzca a las mismas conclusiones.

Si se tiene en cuenta lo planteado en tomo a los problemas del conocimiento que se han analizado, se concluye que no hay transmisión de conocimientos. Tanto quien es el emisor de un conocimiento como el destinatario están atravesados por los conflictos de la sociedad y de la ciencia o disciplinas en que están situados. Sócrates no transmitía un saber: cuestionaba a aquél que creía saber y al saber mismo, el profesor no transmite en términos de vaciar sobre un recipiente una serie de contenidos. El estudiante no está vacío de conocimientos; por el contrario, está lleno de todos aquellos que le han dado el sentido común, las ideologías, los mitos, las leyendas, las culturas. La "llenura de saber" del profesor conduce al autoritarismo, por- que cree saberlo todo y el único parámetro de verdad está dado en el que él impone; por tanto, es excluyente.

La formación de un espíritu crítico y científico obliga a producir una actitud de vida. No se puede pensar en que se tiene un espacio cerrado en el cual se realiza la operatividad científica y crítica y otro en el cual se desenvuelve la acción de nuestra vida, nuestros intereses, nuestros deseos, las formas de nuestra irracionalidad. Es inaceptable pensar estas formas de comportamientos esquizoides; aunque de hecho puede haberlos. Tampoco se puede caer en lo contrario; pretender que se le imponga a la vida una forma de racionalidad que hace de cualquier actitud una acción fría, regulada para no escapar al horizonte del cálculo, de la medida, de la geometrización.

Esta reflexión se hace más importante con seres sociales cuya práctica tiene por objeto la producción o reproducción de saberes. Se puede reducir el trabajo a una simple forma repetitiva de conocimientos que no hace más que convertir en datos cualquier producción de saber. Resultando la doble implicación de consolidar una forma pasiva en la acción pedagógica en cuanto no hay producción, no hay creatividad. Esto propicia en el estudiante la actitud de ser simplemente conocedor de saberes ya constituidos que solo merecen repetirse pero no cuestionarse. Estimula la tara de la interiorización de una especie de división internacional de los saberes, en la cual los países de menor desarrollo económico no podrían producir nuevas formas de pensamiento.

Por lo cual no se puede pretender que la actitud crítica y científica se forma con la simple obtención de manuales o por la claridad que se obtenga 
sobre cómo investigar. En gran parte, es un problema eminentemente práctico que obliga a afrontar las formas y líneas de investigación que se quieran efectuar con placer, con deseo, articulado a un proyecto de vida. Tampoco se puede reducir el problema del conocimiento a la relación entre el sujeto y el objeto. Ni la práctica pedagógica al problema de un sujeto que sabe y otro que no sabe. En ambos casos se trata de condiciones de transformación teórica, cultural, de procesos concretos de producción.

A manera de epílogo se puede concluir invocando los planteamientos de Roland Barthes:

"Hay una edad en la que se enseña lo que se sabe: pero inmediatamente viene otra en la que se enseña lo que no se sabe; eso se llama investigar. Quizá ahora arriba la edad de otra experiencia: la de desaprender, de dejar trabajar a la recomposición imprevisible que el olvido impone a la sedimentación que uno ha atravesado. Esta experiencia creo que tiene un nombre ilustre y pasado de moda que osaré tomar aqui sin complejos, en la encrucijada misma de su etimología: sapientia: ningún poder, un poco de prudente saber y el máximo posible de sabor."13

13 Roland Barthes. Op. cit. p. 150 